\title{
CD47 targeted near-infrared photo-immunotherapy: a promising tool combining monoclonal antibodies and photodynamics for treating human bladder cancer
}

\author{
George Bou Kheir, Fouad Aoun, Thierry Roumeguere \\ Department of Urology, Erasme Hospital, Brussels, Belgium \\ Correspondence to: George Bou Kheir. Urology Resident, Erasme Hospital, Brussels, Belgium. Email: georgeboukheir@live.com; Fouad Aoun. \\ Attending Urologist, Bordet Institute, Brussels, Belgium. Email: fouad.aoun@bordet.be; Thierry Roumeguere. Head of Department, Erasme \\ Hospital, Brussels, Belgium. Email: thierry.roumeguere@erasme.ulb.ac.be. \\ Provenance: This is an invited article commissioned by Section Editor Xiao Li (Department of Urology, Jiangsu Cancer Hospital \& Jiangsu Institute \\ of Cancer Research \& Nanjing Medical University Affiliated Cancer Hospital, Nanjing, China). \\ Comment on: Kiss B, van den Berg NS, Ertsey R, et al. CD47-targeted near-infrared photoimmunotherapy for human bladder cancer. Clin Cancer \\ Res 2019;25:3561-71.
}

Submitted Jun 29, 2019. Accepted for publication Jul 09, 2019.

doi: $10.21037 /$ tau. 2019.07 .10

View this article at: http://dx.doi.org/10.21037/tau.2019.07.10

New technologies are of interest today in diagnosing and treating bladder cancer. Photodynamic diagnosis using violet light after instillation of hexosaminolaevulanic acid (HAL) or 5-aminolaevulanic acid (ALA) has been shown to reduce short term and long term recurrence of bladder cancer according to a systematic review and is now accepted as a diagnostic modality in EAU guidelines (1). However, photodynamic therapy using HAL was not further developed because of lack of specificity and side effects (2). In the same field of physical cancer therapies using electromagnetic wave radiation, nearinfrared photo-immunotherapy (NIR-PIT) conjugates the use of molecular-targeted therapies where a monoclonal antibody is used as a delivery vehicle to a photo-sensitive "nano-dynamite" that activates cytotoxicity under nearinfrared light (3). In comparison with photodynamic therapy, targeting with a monoclonal antibody increases the specificity of NIR-PIT and decreases local side effects.

Kiss et al. assessed the efficacy in vitro and in vivo of CD47-targeted near-infrared photo-immunotherapy in human bladder cancer cells using first, three human bladder cell lines (UMUC-3, HT-1376 and 639-V) and then bladder cancer cells derived from 5 fresh surgical specimens of patients that underwent transurethral resection of a bladder tumor or radical cystectomy, and later in vivo after subcutaneously grafting $639-\mathrm{V}$ cells in NSG mice. Results were promising since death was induced among $90 \%$ of $639 \mathrm{~V}$ and UMUC-3, 50\% among HT-1376, 75\% of 4 cells obtained from 4 patients and $55 \%$ of cells obtained from a T2HG patient. HT-1376 cells are known to have RB-1 mutations. Two of the patients examined exhibited a T2HG bladder tumor and had a different rate of NIR-PIT induced death $(55 \%$ vs. $75 \%)$. This variation in the response may be due to the single nucleotide variation (SNP) in the different bladder tumor cells and further studies may be needed to assess which patient is mostly to benefit from NIR-PIT.

CD47 seems as well an interesting pathway in immunologic responses to cancer as it was shown that the interaction between CD47 and SPR $\alpha$ would activate the "don't eat me" pathway and thus, the blockage of CD47 promotes the phagocytosis of the CD47+ tumor cells and may prevent further metastasis (4). NIR-PIT showed as well an increased expression of calcineutrin, a molecule implicated in the activation of the "eat-me" pathway (5). The CD47-targeted NIR-PIT induction of phagocytosis was established in the study of Kiss et al. where the results have shown a significantly increased in vitro phagocytic activity and a trend to an increased influx of macrophages in vivo by immunohistochemistry on tumor tissue samples from the xenograft models. The combined functions of CD47-targeted NIR-PIT enhancement of tumor cytotoxicity and induction of direct cell death could have 
an impact on radiation dose reduction specially that skin damage was observed with the weekly radiation regimen which had led to a modification of the protocol.

Monoclonal antibodies offer significant advantages because of their protein structure and biochemical properties but also some disadvantages such as their route of administration (only intraperitoneal or intravenous). As for the limitations of this study, we noted the use of a nonorthotopic model where the tumor cells are grafted in the back of the NSG mice but not in the bladder which might be ideal for future implications in clinical trials. The tumor microenvironment has to be considered much different subcutaneously and within the bladder. Intra-vesical therapy is not as straight forward and attempts were made with failure. In a study assessing EGFR-targeted NIR-PIT, UMUC-5 and other cell lines were inoculated intravesically in nude mice to establish orthotopic intra-vesical human tumors, the tumor formation rate was $<10 \%$ and in animals where tumor developed, it spontaneously regressed (6).

In today's armamentarium to treat bladder cancer, immunotherapy has provided a wealth of new treatment options alongside surgery, radiation and chemotherapy. Targeting CD47 axis is one hotspot in cancer therapy. There are now many anti-CD47 antibodies and fusion proteins in clinical trials and some other agents in preclinical investigation. The ubiquitous expression of CD47 in normal tissues rise concerns about potential side effects for off-target cytotoxicity (7). However, the particularity of bladder tumors is the possibility of being targeted endoscopically, thus bypassing the circulation, specially that CD47 is expressed in both NMIBC and MIBC, but absent on the terminally differentiated luminal umbrella cells of normal human urothelium (8). Coupled to NIR-PIT, it provides a potential treatment in an officebased setting. Clinical trials are awaited to further assess its efficacy, and to find the best patients who could benefit from this treatment modality.

\section{Acknowledgments}

None.

Cite this article as: Bou Kheir G, Aoun F, Roumeguere T. CD47 targeted near-infrared photo-immunotherapy: a promising tool combining monoclonal antibodies and photodynamics for treating human bladder cancer. Transl Androl Urol 2019;8(6):779-780. doi:10.21037/tau.2019.07.10

\section{Footnote}

Conflicts of Interest: The authors have no conflicts of interest to declare.

Ethical Statement: The authors are accountable for all aspects of the work in ensuring that questions related to the accuracy or integrity of any part of the work are appropriately investigated and resolved.

\section{References}

1. Babjuk M, Burger M, Compérat E, et al. EAU guidelines on non-muscle-invasive bladder cancer (TaT1 and CIS). Available online: https://uroweb.org/wp-content/ uploads/05-Non-muscle-Invasive-BC_TaT1_2019v2.pdf (Accessed 31 May 2019).

2. Bader MJ, Stepp H, Beyer W, et al. Photodynamic therapy of bladder cancer - a phase I study using hexaminolevulinate (HAL). Urol Oncol 2013;31:1178-83.

3. Mitsunaga M, Ogawa M, Kosaka N, et al. Cancer cellselective in vivo near infrared photoimmunotherapy targeting specific membrane molecules. Nat Med 2011;17:1685-91.

4. Horrigan SK, Reproducibility Project: Cancer Biology. Replication study: the CD47-signal regulatory protein alpha (SIRPa) interaction is a therapeutic target for human solid tumors. eLife 2017;6:e18173.

5. Ogawa M, Tomita Y, Nakamura Y, et al. Immunogenic cancer cell death selectively induced by near infrared photoimmunotherapy initiates host tumor immunity. Oncotarget 2017;8:10425-36.

6. Railkar R, Krane LS, Li QQ, et al. Epidermal growth factor receptor (EGFR)-targeted photoimmunotherapy (PIT) for the treatment of EGFR-expressing bladder cancer. Mol Cancer Ther 2017;16:2201-14.

7. Zhang X, Fan J, Ju D. Insights into CD47/SIRP $\alpha$ axistargeting tumor immunotherapy. Antib Ther. 2018;1:27-32.

8. Pan Y, Volkmer JP, Mach KE, et al. Endoscopic molecular imaging of human bladder cancer using a CD47 antibody. Sci Transl Med 2014;6:260ra148. 